\title{
BMI Modulates the Effect of Thyroid Hormone on Lipid Profile in Euthyroid Adults
}

\author{
Yanqiu Wang, ${ }^{1}$ Qinglei Yin, ${ }^{1}$ Min Xu, ${ }^{1}$ Qicheng Ni, ${ }^{1}$ Weiqing Wang, ${ }^{1}$ and Qidi Wang ${ }^{1,2}$ \\ ${ }^{1}$ Shanghai National Clinical Research Center for Endocrine and Metabolic Diseases, Key Laboratory for Endocrine and Metabolic \\ Diseases of Chinese Health Ministry, Ruijin Hospital Affiliated to Shanghai Jiaotong University School of Medicine, Shanghai \\ 200025, China \\ ${ }^{2}$ Sino-French Research Center for Life Sciences and Genomics, Ruijin Hospital Affiliated to Shanghai Jiaotong University School of \\ Medicine, Shanghai 200025, China
}

Correspondence should be addressed to Qidi Wang; qidiwang@yahoo.com

Received 30 March 2017; Revised 26 May 2017; Accepted 8 June 2017; Published 13 August 2017

Academic Editor: Constantinos Pantos

Copyright (C) 2017 Yanqiu Wang et al. This is an open access article distributed under the Creative Commons Attribution License, which permits unrestricted use, distribution, and reproduction in any medium, provided the original work is properly cited.

\begin{abstract}
The impacts of thyroid hormones (TH) on lipid profile in euthyroid adults have gained much attention. It is currently unknown whether BMI influences such interaction. In the present study, we investigate the role of BMI in modulating the association between TH and lipid parameters in 1372 euthyroid healthy adults. Our results show that thyroid parameters are differentially associated with lipid profile. FT3 is positively correlated with total cholesterol $(\beta=0.176 \pm 0.046, P<0.001)$ and LDL cholesterol levels $(\beta=0.161 \pm 0.040, P<0.001)$. FT4 is negatively correlated with TG $(\beta=-0.087 \pm 0.029, P<0.01)$ while positively correlated with HDL cholesterol levels $(\beta=0.013 \pm 0.005, P<0.01)$. TSH is positively associated with TG $(\beta=0.145 \pm 0.056$, $P<0.05)$ and total cholesterol levels $(\beta=0.094 \pm 0.030, P<0.01)$. Importantly, BMI modulates the effect of TH on lipid profile: the interaction of FT4 and BMI and the interaction of FT3 and BMI reach statistical significance in predicting TG and HDL cholesterol levels, respectively. Stratified according to BMI levels, most associations between $\mathrm{TH}$ and lipid profile are significant only in normal-weight group. In conclusion, in euthyroid adults, high normal FT3, TSH levels, and low normal FT4 levels are associated with unfavorable lipid profile. BMI mediates the effect of thyroid function on lipid profile in euthyroid adults.
\end{abstract}

\section{Introduction}

Dyslipidemia constitutes a major risk factor for premature atherosclerosis and cardiovascular disease (CVD), which is a major cause of morbidity and mortality in both developed and developing countries [1]. Dyslipidemia is defined as increased triglycerides (TG) or low-density lipoprotein cholesterol (LDL cholesterol) levels, or decreased highdensity lipoprotein cholesterol (HDL cholesterol) levels. A clustering of risk factors for developing dyslipidemia is age, eating habits, physical activity, stress, and heredity, as well as thyroid function [2].

Thyroid hormones $(\mathrm{TH})$ regulate multiple metabolic processes [3]. It is widely accepted that overt thyroid dysfunctions (both hypo- and hyperthyroidism) are correlated with alternations in lipid and glucose metabolism [2].
Hyperthyroidism leads to a hypermetabolic state and increased lipolysis, which is characterized by weight loss, lower plasma levels of HDL cholesterol, LDL cholesterol, and leptin [2]. Conversely, hypothyroidism has the opposite effects: hypothyroid patients are presented with higher plasma TG, total cholesterol, and LDL cholesterol levels $[2,4,5]$. The mechanisms of thyroid hormone on metabolic phenotype in patients with thyroid disease have been extensively investigated $[3,5]$. Recently, the impacts of thyroid hormones within normal ranges on lipid profile have also been clarified [6-10]. In euthyroid adults, FT3 within normal range is positively correlated to dyslipidemia: high normal FT3 is associated with a less favorable metabolic phenotype in pregnant women [10] and in general euthyroid population [6]. On the other hand, several studies have shown that low normal FT4 or high normal TSH levels are associated with unfavorable lipid profile 
and higher CVD risk [7, 9, 11-14]. Roos et al. found a negative relation between FT4 and plasma total cholesterol and LDL cholesterol levels [7]. Moreover, a negative association between FT4 and TG was also reported in euthyroid adults, even after adjustment for potential confounders [15, 16]. TSH was positively associated with total cholesterol and LDL cholesterol levels both in euthyroid men and women $[11,13]$. Interestingly, in obese euthyroid population, Temizkan et al. could not identify significant associations between TSH and atherogenic dyslipidemia [8]. Moreover, in the obese setting, Marzullo et al. reported that TSH was positively associated with total cholesterol levels; however, the association disappeared after controlling for individuals' confounders, including BMI [16]. It is known that thyroid function status, even within normal ranges, might correlate with alternations in body weight and energy metabolism [17-19]. Moreover, it is well documented that obesity is correlated not only with unfavorable metabolic profiles but also with changes in thyroid hormone levels [20]. So far, little is known whether BMI mediates the influence of thyroid hormones on lipid profile in euthyroid subjects.

The objective of the current study is to examine the association between thyroid hormones and lipid parameters in euthyroid adults. Furthermore, the role of BMI in modulating the association between thyroid hormones and lipid profile is determined.

\section{Methods}

2.1. Study Population. We conducted this retrospective study with subjects who participated in a routine health screening examination in Ruijin Hospital affiliated to Shanghai Jiao Tong University School of Medicine between 2007 and 2010. Individuals with previously diagnosed chronic cardiovascular diseases, diabetes mellitus, and liver and kidney diseases were excluded from our study. Women with diagnosed pregnancy were also excluded. Furthermore, individuals receiving thyroid hormone substitutions or antithyroid drugs, which may potentially interfere with thyroid function or having TPO antibodies, antithyroglobulin antibodies above the clinical cutoff for positivity, or with TSH levels outside the normal reference range of our laboratory (TSH: $0.35-4.94 \mathrm{mIU} / \mathrm{L})$, were also excluded from our analyses, finally leaving 1372 subjects for inclusion in this investigation.

The study was approved by the Medical Ethics Committee of Ruijin Hospital, Shanghai Jiao Tong University School of Medicine and was conducted in accord with institutional guidelines. The study was in accordance with the principle of the Helsinki Declaration II. All study participants provided written informed consents.

2.2. Measurements. Data on gender and age were obtained from all the subjects. Standing height $(\mathrm{cm})$ was measured using a wall-mounted Harpenden Stadiometer. Body weight $(\mathrm{kg})$ was measured in light indoor clothing without shoes. Body mass index is defined as the body weight (kg) divided by the square of the body height $(\mathrm{m})$.
Normal weight is defined as $18.5 \leq \mathrm{BMI}<25 \mathrm{~kg} / \mathrm{m}^{2}$, while overweight and obese are defined as BMI $\geq 25 \mathrm{~kg} / \mathrm{m}^{2}$ according to the WHO criteria [21].

2.3. Biochemical Determinations. Blood samples were obtained from all the subjects after overnight fasting. Chemiluminescent methods (Cobas E601; Roche, Basel, Switzerland) were used to quantitate thyroid function based on free triiodothyronine $\left(\mathrm{FT}_{3}\right)$, free thyroxine $\left(\mathrm{FT}_{4}\right)$, and thyrotropin (TSH) levels. Serum total cholesterol, low-density lipoprotein cholesterol (LDL cholesterol), high-density lipoprotein cholesterol (HDL cholesterol), and TG were measured using an autoanalyzer (ARCHITECT ci16200 analyzer; Abbott Laboratories, Abbott, IL) in the Shanghai Institute of Endocrine and Metabolic Diseases.

2.4. Statistical Analysis. All statistical analyses (linear regression analyses, logistic regression analyses, and one-way analysis of variance (ANOVA)) were performed using SAS 9.2 (SAS Institute, Cary, NC). Data were given as mean \pm standard deviation for normally distributed continuous parameters, and for skewness distribution data, median and interquartile range was used. Descriptive statistics were used to describe the study population at the baseline.

Linear regression analysis was used to identify the association between different thyroid parameters and different lipid parameters. These models were all adjusted for age, gender, and BMI. Each BMI subgroup was sequentially assigned to tertiles according to their plasma FT3, FT4, and TSH levels. Associations between thyroid function parameters and lipid profile among tertiles were identified using oneway ANOVA in each BMI subgroup. $P$ for interaction resulted from linear regression analysis with FT3*BMI (FT4 ${ }^{*} \mathrm{BMI}$, or $\left.\mathrm{TSH}^{*} \mathrm{BMI}\right)$ as independent variable and lipid profile as dependent variables, after multiple adjustment for age, gender, BMI, and FT3 (or FT4 and TSH). We further divided the population into subgroups according to gender, and $P$ for interaction was calculated after adjustment for age, BMI, and FT3 (or FT4 and TSH).

The $P$ values reported were two sided. Statistical significance was assumed for $P$ values $<0.05$.

\section{Results}

3.1. General Characteristics of the Study Population. Characteristics of the study population, their anthropometric data, metabolic parameters, and descriptions for thyroid hormones were shown in Table 1. A total of 1372 euthyroid subjects (973 males and 399 females) were enrolled in this study, in which $36.0 \%$ were overweight $\left(25 \leq \mathrm{BMI}<30 \mathrm{~kg} / \mathrm{m}^{2}\right)$ and $4.2 \%$ were obese $\left(\mathrm{BMI} \geq 30 \mathrm{~kg} / \mathrm{m}^{2}\right)$. There were evident differences in lipid parameters and thyroid hormone levels between subgroups with $\mathrm{BMI} \geq 25 \mathrm{~kg} / \mathrm{m}^{2}$ (overweight/obese group: average BMI $\left.27.28 \pm 1.96 \mathrm{~kg} / \mathrm{m}^{2}\right)$ and with $18.5 \leq \mathrm{BMI}<25 \mathrm{~kg} / \mathrm{m}^{2}$ (normal-weight group: average BMI $22.43 \pm 1.70 \mathrm{~kg} / \mathrm{m}^{2}$ ). Subjects in the overweight/obese group had worse lipid profile compared to the normal-weight controls: they had significantly higher TG levels $(1.90$ (1.32-2.86) versus $1.27(0.87-1.89) \mathrm{mmol} / \mathrm{L}, P<0.001)$, total 
TABle 1: General, anthropometric, hormonal, and metabolic parameters of the study population.

\begin{tabular}{|c|c|c|c|}
\hline & $\begin{array}{c}18.5 \leq \mathrm{BMI}<25 \\
(n=820)\end{array}$ & $\begin{array}{l}\mathrm{BMI} \geq 25 \\
(n=552)\end{array}$ & $P$ value \\
\hline \multicolumn{4}{|l|}{ General characteristics } \\
\hline Age (years) & $48.33 \pm 9.28$ & $48.64 \pm 8.34$ & 0.525 \\
\hline Height $(\mathrm{cm})$ & $167.56 \pm 7.05$ & $170.61 \pm 6.08$ & $<0.001^{* * *}$ \\
\hline Body weight $(\mathrm{kg})$ & $63.20 \pm 8.00$ & $79.52 \pm 8.28$ & $<0.001^{* * *}$ \\
\hline BMI $\left(\mathrm{kg} / \mathrm{m}^{2}\right)$ & $22.43 \pm 1.70$ & $27.28 \pm 1.96$ & $<0.001^{* * *}$ \\
\hline \multicolumn{4}{|l|}{ Lipid profile } \\
\hline $\mathrm{TG}(\mathrm{mmol} / \mathrm{L})$ & $1.27(0.87-1.89)$ & $1.90(1.32-2.86)$ & $<0.001^{* * *}$ \\
\hline Total cholesterol $(\mathrm{mmol} / \mathrm{L})$ & $4.82 \pm 0.96$ & $4.95 \pm 0.94$ & $<0.05^{*}$ \\
\hline HDL cholesterol (mg/L) & $13.21 \pm 3.34$ & $11.64 \pm 2.95$ & $<0.001^{* * *}$ \\
\hline LDL cholesterol $(\mathrm{mg} / \mathrm{L})$ & $29.53 \pm 8.18$ & $29.93 \pm 8.18$ & 0.386 \\
\hline \multicolumn{4}{|l|}{ TSH and thyroid hormones } \\
\hline FT3 (pmol/L) & $4.11 \pm 0.57$ & $4.29 \pm 0.58$ & $<0.001^{* * *}$ \\
\hline FT4 (pmol/L) & $12.62 \pm 1.70$ & $12.41 \pm 1.54$ & $<0.05^{*}$ \\
\hline TSH (mIU/L) & $1.66 \pm 0.86$ & $1.58 \pm 0.85$ & 0.074 \\
\hline
\end{tabular}

Subjects on thyroid medications, with TPO antibodies, anti-thyroglobulin antibodies above clinical cutoff, and with TSH levels outside the reference range, were excluded from further analyses. We stratified the study group according to BMI levels: $18.5 \leq \mathrm{BMI}<25 \mathrm{~kg} / \mathrm{m}^{2}$ : normal-weight group; $\mathrm{BMI} \geq 25 \mathrm{~kg} / \mathrm{m}^{2}$ : overweight/obese group. All continuous parameters were presented as mean \pm standard deviation. For skewness distribution data, median and interquartile range was used. $P$ values were calculated from one-way analysis of variance. BMI: body mass index; FT3: free triiodothyronine; FT4: free thyroxine; TSH: thyrotropin; TG: triglycerides; HDL: high-density lipoprotein; LDL: low-density lipoprotein. Significant associations are indicated in bold. $P<0.05$ was considered statistically significant. ${ }^{*} P<0.05,{ }^{* * *} P<0.001$.

cholesterol levels $(4.95 \pm 0.94$ versus $4.82 \pm 0.96 \mathrm{mmol} / \mathrm{L}$, $P<0.05)$, and lower HDL cholesterol levels $(11.64 \pm 2.95$ versus $13.21 \pm 3.34 \mathrm{mg} / \mathrm{L}, P<0.001$ ) (Table 1 ). There was no significant difference in LDL cholesterol levels between the two groups (Table 1).

Thyroid hormone levels between subjects in different BMI categories also varied significantly. Subjects in the overweight/obese group showed increased FT3 levels $(4.29 \pm 0.58$ versus $4.11 \pm 0.57 \mathrm{pmol} / \mathrm{L}, P<0.001)$, but decreased FT4 levels $(12.41 \pm 1.54$ versus $12.62 \pm 1.70 \mathrm{pmol} / \mathrm{L}, \quad P<0.05)$. Moreover, TSH levels were slightly lower in the overweight/ obese group but did not reach statistical significance $(1.58 \pm 0.85$ versus $1.66 \pm 0.86 \mathrm{mIU} / \mathrm{L}, P=0.074)$.

3.2. The Association between TH and Lipid Profile in Euthyroid Adults. To understand the relationship between the levels of thyroid hormones and lipid parameters, we performed linear regression analyses, adjusted for age, gender, and BMI in euthyroid subjects. As shown in Table 2, FT3 and FT4 were differentially associated with lipid parameters in euthyroid subjects. FT3 was significantly and positively correlated with total cholesterol $(\beta=0.176 \pm 0.046, P<0.001)$ and LDL cholesterol concentrations $(\beta=0.161 \pm 0.040, \quad P<0.0001)$. On the contrary, FT4 levels were negatively correlated with TG $(\beta=-0.087 \pm 0.029, \quad P<0.01)$ but positively associated with $\mathrm{HDL}$ cholesterol levels $(\beta=0.013 \pm 0.005, P<0.01)$.
TSH levels were significantly and positively related to both TG $(\beta=0.145 \pm 0.056, P<0.05)$ and total cholesterol levels $(\beta=0.094 \pm 0.030, P<0.01)$.

3.3. BMI Modulates the Association between TH and Lipid Profile in Euthyroid Adults. We further tested whether there were interactions between BMI and thyroid parameters in modulating lipid profile. Importantly, the interaction of FT3 and BMI and the interaction of FT4 and BMI reached statistical significance in predicting serum HDL cholesterol $(P<0.05)$ and TG concentrations $(P<0.05)$, respectively, after multiple adjustment for age, gender, BMI, and FT3 (or FT4) (Table 3). We also examined the interaction of TSH and BMI in predicting lipid parameters; however, we could not find statistical significance in predicting any of the parameters in the whole population (Table 3 ).

It is known that thyroid function and lipid profile can be influenced by gender $[6,12,14]$. Therefore, gender-based interaction analyses were also performed. As shown in Table S1 available online at https://doi.org/10.1155/2017/8591986, the interaction of FT3 and BMI remained significant in predicting serum HDL cholesterol in male subjects $(P<0.05)$, whereas the interaction of FT4 and BMI was significant in modulating TG concentrations in females $(P<0.05)$. Interestingly, the interaction of TSH and BMI also appeared statistical significance in both male and female subjects: predicting total cholesterol and LDL cholesterol levels in males (both $P<0.05$ ), while predicting TG levels in females $(P<0.01)$ (Table S1).

3.4. The Association between TH and Lipid Profile in the Normal-Weight and Overweight/Obese Groups. Since BMI modulates the interaction between thyroid parameters and lipid profile, we further checked whether the association between thyroid parameters and lipid profile varied in subjects with different BMI. We performed linear regression analyses separately in the normal-weight and overweight/ obese groups (Table 3). In the normal-weight and overweight/obese groups, the subjects were further sequentially assigned to tertiles according to their plasma FT3 (Table 4), FT4 (Table 5), or TSH (Table 6) levels.

A positive correlation between FT3 and TG levels was only significant in the normal-weight subjects $(\beta=0.145 \pm 0.071$, $P<0.05$; Table 3), with an increase in TG level across the tertiles $(P<0.05$; Table 4$)$. In both normal-weight and overweight/obese groups, FT3 was positively associated with total cholesterol and LDL cholesterol levels (both $P<0.01$, Table 3). In parallel, the higher FT3 tertiles (T2 and T3) had significantly elevated total cholesterol $(P<0.01)$ and LDL cholesterol levels $(P<0.01)$ compared to the lowest tertile (T1) in both normal-weight and overweight/obese groups (Table 4).

We then stratified the population according to their serum FT4 levels. A significant correlation between FT4 and TG $(\beta=-0.071 \pm 0.022, P<0.01)$ as well as FT4 with HDL $(\beta=0.017 \pm 0.006, P<0.01)$ were only detected in the normal-weight subjects (both $P<0.01$ ), but not in the overweight/obese group (Table 3). Similarly, significantly decreased plasma TG and increased HDL cholesterol levels 
TABLE 2: The association between lipid profile and thyroid parameters.

\begin{tabular}{|c|c|c|c|c|c|c|}
\hline \multirow{2}{*}{ Dependent variable } & \multicolumn{2}{|c|}{ FT3 } & \multicolumn{2}{|c|}{ FT4 } & \multicolumn{2}{|c|}{ TSH } \\
\hline & $\beta \pm \mathrm{SE}$ & $P$ & $\beta \pm \mathrm{SE}$ & $P$ & $\beta \pm \mathrm{SE}$ & $P$ \\
\hline TG & $0.075 \pm 0.087$ & 0.392 & $-0.087 \pm 0.029$ & $0.003^{* *}$ & $0.145 \pm 0.056$ & $0.010^{*}$ \\
\hline $\mathrm{CHOL}$ & $0.176 \pm 0.046$ & $0.0002^{* * *}$ & $0.005 \pm 0.016$ & 0.761 & $0.094 \pm 0.030$ & $0.002^{* *}$ \\
\hline HDL-C & $0.029 \pm 0.015$ & 0.051 & $0.013 \pm 0.005$ & $0.007^{* *}$ & $-0.002 \pm 0.010$ & 0.825 \\
\hline LDL-C & $0.161 \pm 0.040$ & $P<0.0001^{* * *}$ & $0.016 \pm 0.013$ & 0.237 & $0.039 \pm 0.026$ & 0.137 \\
\hline
\end{tabular}

Reported values are betas \pm standard error and results from linear regression analysis with lipid parameters as dependent variables and thyroid parameters as independent variables. Betas are scaled and adjusted for age, gender, and BMI. CHOL: total cholesterol; HDL-C: high-density lipoprotein cholesterol; LDL-C: low-density lipoprotein cholesterol. Significant associations are indicated in bold. $P<0.05$ was considered statistically significant. ${ }^{*} P<0.05$, ${ }^{* *} P<0.01$, and ${ }^{* * *} P<0.001$.

(both $P<0.01)$ were observed in the highest tertile of FT4 only in the normal-weight group (Table 5).

A significant positive association between serum TSH levels and total cholesterol levels was only found in the normal-weight group $(\beta=0.110 \pm 0.039, P<0.01$; Table 3$)$. Indeed, significantly increased total cholesterol levels in the highest tertile of TSH were observed exclusively in the normal-weight group $(P<0.01$; Table 6$)$.

\section{Discussion}

Thyroid hormones influence key metabolic pathways which control energy balance by regulating energy storage and expenditure [5]. Overt thyroid dysfunctions are associated with alternations in lipid metabolism [2-5]. Recent studies have shown that changes in thyroid hormone levels, even within the physiological ranges, may contribute to the deteriorating of atherogenic lipid profile $[6,7,9,11,12]$. The present study with 1372 euthyroid adults demonstrated that FT3 was significantly and positively correlated with total and LDL cholesterol concentrations, whereas FT4 levels were negatively correlated with TG but positively associated with HDL cholesterol levels. Moreover, TSH levels were significantly and positively related to both TG and total cholesterol levels. Our findings were in line with several previous studies $[6,7,12-16,22,23]$, showing that low normal FT4 [7, 9, 11-14] and high normal FT3 [6, 10] and TSH $[11,13]$ levels are associated with unfavorable lipid profile in euthyroid subjects. However, in obese euthyroid population, previous studies showed that some of these associations could not be identified [8] or disappeared after controlling for individuals' confounders including BMI [16]. Interestingly, when we performed linear regression analyses separately in the normal-weight and overweight/obese groups, we clearly reported that the associations between thyroid parameters and lipid parameters were not identical: the correlation between FT3 (or FT4) and TG and the correlation between TSH and total cholesterol were only significant in the normal-weight group, but not in individuals with $\mathrm{BMI} \geq 25 \mathrm{~kg} / \mathrm{m}^{2}$. The observations strongly indicate that body weight might interfere with the effect of $\mathrm{TH}$ on lipid profile.

The thyroid and adipose tissue are organs producing thyroid hormones and adipokines, respectively [24]. Both of them play central roles in the metabolism of the body. Several studies have shown that variations in TSH and thyroid hormones within normal ranges can influence body weight and subsequently alter lipid profile [25]. On the other hand, disturbed TH levels have been reported in obese subjects [25] and were also observed in subjects with $\mathrm{BMI} \geq 25 \mathrm{~kg} / \mathrm{m}^{2}$ in the current study. However, the influence of body weight on the interaction of TH and lipid profile has not been clearly clarified in the literature. Importantly, we showed that serum FT4 levels and BMI had interactions in predicting serum TG levels, whereas FT3 and BMI had interactions in predicting HDL cholesterol levels in euthyroid population. Such interactions remained significant and even more pronounced when we performed gender-based interaction analyses: TSH and BMI reached significance in predicting total cholesterol and LDL cholesterol levels in males, as well as TG levels in females.

As generally accepted, obesity is closely associated with dyslipidemia, manifested as elevated fasting and postprandial TG in combination with the preponderance of small dense LDL and low HDL cholesterol [26]. Obesity increases free fatty acid (FFA) fluxes to the liver, adipose tissue, and skeletal muscle, which leads to alter expression of lipoprotein lipase (LPL) activity and hampers lipolysis and TG accumulation and transport, subsequently causes dyslipidemia [26]. It is currently unknown how BMI interferes with the effect of thyroid hormones on lipid profile, and it is possible that certain adipokines, such as leptin, play a role via hypothalamicpituitary-thyroid axis $[27,28]$. Although changes in $\mathrm{TH}$ levels within normal ranges have independent influence on lipid profile in normal-weight subjects, such impact might be neglectable in overweight/obese individuals. Thus, the current observations might be clinical relevant regarding strategies for individualized lipid control. In normal-weight subjects, TH levels within normal ranges should be taken into account: low normal FT3, TSH levels, and high normal FT4 levels might be beneficial for lipid control. However, in overweight/obese subjects, weight loss absolutely takes priority. The disturbed levels of TH in obesity can be reversed after weight loss [29] and might have additional impact on lipid management.

The novelty of the study is that, to the best of our knowledge, we are the first to identify the role of BMI in modulating the effect of thyroid hormones on lipid profile in euthyroid adults. However, our study has several limitations. First, due to the small sample size of obese 


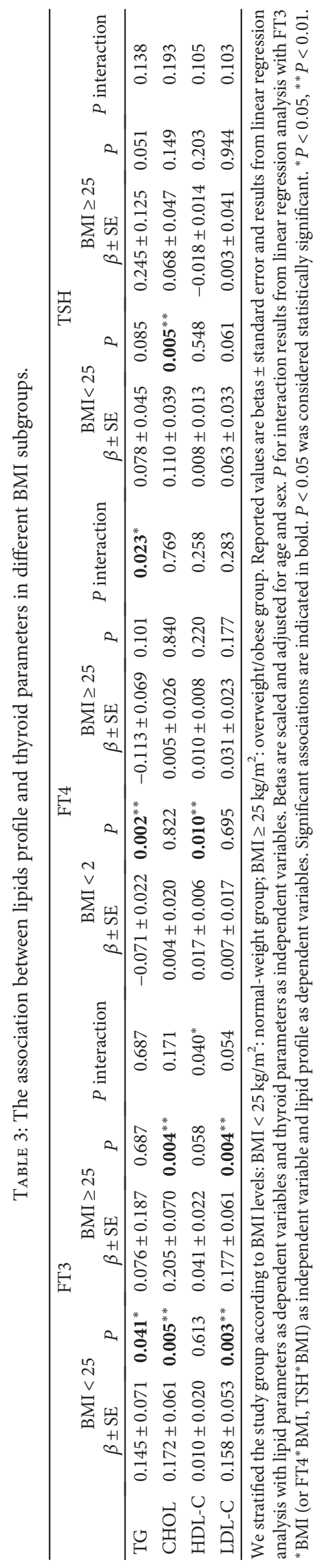


TABLE 4: Lipid profile in different FT3 and BMI categories.

\begin{tabular}{lcccccccc}
\hline & \multicolumn{3}{c}{ BMI $<25$} & & \multicolumn{3}{c}{ BMI $\geq 25$} \\
& Group 1 & Group 2 & Group 3 & $P$ & Group 4 & \multicolumn{2}{c}{ Group 5 } & Group 6 \\
& FT3 T1 & FT3 T2 & FT3 T3 & & FT3 T1 & FT3 T2 & FT3 T3 \\
\hline TG & $1.05(0.74-1.56)$ & $1.29(0.90-1.84)$ & $1.55(1.02-2.17)$ & $\mathbf{0 . 0 4 1}^{*}$ & $1.75(1.13-2.60)$ & $1.88(1.34-2.90)$ & $2.09(1.40-3.06)$ & 0.687 \\
CHOL & $4.72 \pm 0.96$ & $4.81 \pm 0.95$ & $4.92 \pm 0.95$ & $\mathbf{0 . 0 0 5}^{* *}$ & $4.82 \pm 0.87$ & $4.97 \pm 0.98$ & $5.06 \pm 0.94$ & $\mathbf{0 . 0 0 4} 4^{* *}$ \\
HDL-C & $1.36 \pm 0.33$ & $1.32 \pm 0.34$ & $1.28 \pm 0.32$ & 0.613 & $1.16 \pm 0.31$ & $1.14 \pm 0.28$ & $1.19 \pm 0.30$ & 0.058 \\
LDL-C & $2.86 \pm 0.81$ & $2.93 \pm 0.81$ & $3.06 \pm 0.83$ & $\mathbf{0 . 0 0 3}^{* *}$ & $2.85 \pm 0.77$ & $3.03 \pm 0.86$ & $3.09 \pm 0.82$ & $\mathbf{0 . 0 0 4} 4^{* *}$ \\
\hline
\end{tabular}

We stratified the study group according to BMI levels: BMI $<25 \mathrm{~kg} / \mathrm{m}^{2}:$ normal-weight group; BMI $\geq 25 \mathrm{~kg} / \mathrm{m}^{2}$ : overweight/obese group. Then, we stratified each group according to serum FT3 levels; and all continuous parameters were presented as mean \pm standard deviation, after adjustment for age and gender. For skewness distribution data, median and interquartile range was used. $P$ values were calculated from ANOVA (analysis of variance, between categories), after adjustment for age and gender. Significant associations are indicated in bold. $P<0.05$ was considered statistically significant. ${ }^{*} P<0.05,{ }^{* *} P<0.01$.

TABLE 5: Lipid profile in different FT4 and BMI categories.

\begin{tabular}{|c|c|c|c|c|c|c|c|c|}
\hline & \multicolumn{4}{|c|}{$\mathrm{BMI}<25$} & \multicolumn{4}{|c|}{$\mathrm{BMI} \geq 25$} \\
\hline & $\begin{array}{l}\text { Group } 1 \\
\text { FT4 T1 }\end{array}$ & $\begin{array}{l}\text { Group } 2 \\
\text { FT4 T2 }\end{array}$ & $\begin{array}{l}\text { Group } 3 \\
\text { FT4 T3 }\end{array}$ & $P$ & $\begin{array}{l}\text { Group } 4 \\
\text { FT4 T1 }\end{array}$ & $\begin{array}{l}\text { Group } 5 \\
\text { FT4 T2 }\end{array}$ & $\begin{array}{l}\text { Group } 6 \\
\text { FT4 T3 }\end{array}$ & $P$ \\
\hline TG & $1.30(0.93-2.02)$ & $1.36(0.92-1.90)$ & $1.16(0.82-1.71)$ & $0.002^{* *}$ & $2.04(1.36-3.11)$ & $2.18(1.41-3.29)$ & $1.68(1.20-2.35)$ & 0.101 \\
\hline $\mathrm{CHOL}$ & $4.80 \pm 0.95$ & $4.81 \pm 0.92$ & $4.84 \pm 1.00$ & 0.822 & $4.92 \pm 0.96$ & $5.01 \pm 0.88$ & $4.92 \pm 0.96$ & 0.840 \\
\hline HDL-C & $1.30 \pm 0.33$ & $1.31 \pm 0.32$ & $1.35 \pm 0.34$ & $0.010^{* *}$ & $1.14 \pm 0.26$ & $1.14 \pm 0.30$ & $1.22 \pm 0.32$ & 0.220 \\
\hline LDL-C & $2.94 \pm 0.80$ & $2.95 \pm 0.78$ & $2.98 \pm 0.88$ & 0.695 & $2.96 \pm 0.85$ & $2.97 \pm 0.78$ & $3.04 \pm 0.82$ & 0.177 \\
\hline
\end{tabular}

We stratified the study group according to BMI levels: BMI $<25 \mathrm{~kg} / \mathrm{m}^{2}:$ normal-weight group; BMI $\geq 25 \mathrm{~kg} / \mathrm{m}^{2}:$ overweight/obese group. Then, we stratified each group according to serum FT4 levels; all continuous parameters were presented as mean \pm standard deviation, after adjustment for age and gender. For skewness distribution data, median and interquartile range was used. $P$ values were calculated from ANOVA (analysis of variance, between categories), after adjustment for age and gender. Significant associations are indicated in bold. $P<0.05$ was considered statistically significant. ${ }^{* *} P<0.01$.

TABLE 6: Lipid profile in different TSH and BMI categories.

\begin{tabular}{|c|c|c|c|c|c|c|c|c|}
\hline & \multicolumn{4}{|c|}{$\mathrm{BMI}<25$} & \multicolumn{4}{|c|}{$\mathrm{BMI} \geq 25$} \\
\hline & $\begin{array}{l}\text { Group } 1 \\
\text { TSH T1 }\end{array}$ & $\begin{array}{l}\text { Group } 2 \\
\text { TSH T2 }\end{array}$ & $\begin{array}{l}\text { Group } 3 \\
\text { TSH T3 }\end{array}$ & $P$ & $\begin{array}{l}\text { Group } 4 \\
\text { TSH T1 }\end{array}$ & $\begin{array}{l}\text { Group } 5 \\
\text { TSH T2 }\end{array}$ & $\begin{array}{l}\text { Group } 6 \\
\text { TSH T3 }\end{array}$ & $P$ \\
\hline TG & $1.21(0.82-1.88)$ & $1.32(0.93-1.95)$ & $1.23(0.88-1.84)$ & 0.085 & $1.30(1.81-2.76)$ & $1.86(1.27-2.66)$ & $2.01(1.37-3.18)$ & 0.051 \\
\hline CHOL & $4.71 \pm 0.90$ & $4.81 \pm 0.95$ & $4.93 \pm 1.01$ & $0.005^{* *}$ & $4.87 \pm 0.96$ & $4.91 \pm 0.89$ & $5.08 \pm 0.94$ & 0.149 \\
\hline HDL-C & $1.31 \pm 0.32$ & $1.31 \pm 0.34$ & $1.35 \pm 0.34$ & 0.548 & $1.15 \pm 0.30$ & $1.19 \pm 0.29$ & $1.15 \pm 0.30$ & 0.203 \\
\hline LDL-C & $2.88 \pm 0.76$ & $2.96 \pm 0.82$ & $3.03 \pm 0.87$ & 0.061 & $2.96 \pm 0.83$ & $3.01 \pm 0.77$ & $3.00 \pm 0.86$ & 0.944 \\
\hline
\end{tabular}

We stratified the study group according to BMI levels: $\mathrm{BMI}<25 \mathrm{~kg} / \mathrm{m}^{2}$; normal-weight group; BMI $\geq 25 \mathrm{~kg} / \mathrm{m}^{2}$ : overweight/obese group. Then, we stratified each group according to serum TSH levels; all continuous parameters were presented as mean \pm standard deviation, after adjustment for age and gender. For skewness distribution data, median and interquartile range was used. $P$ values were calculated from ANOVA (analysis of variance, between categories), after adjustment for age and gender. Significant associations are indicated in bold. $P<0.05$ was considered statistically significant. ${ }^{* *} P<0.01$.

population, we could not compare overweight and obese subjects separately in our analyses. Second, lacking of smoking status information impeded us to identify the effect of smoking habit on such relation. Third, it would be ideal to initially exclude the subjects on lipid-lowering therapy, although we estimate that this percentage is very low in our study population. Fourth, as a cross-sectional study, the causal relationship could not be inferred. Further studies are needed to clarify whether adjusting certain $\mathrm{TH}$ parameters in euthyroid normal-weight subjects do bring benefits in maintaining lipid homeostasis.

In conclusion, in euthyroid adults, low normal FT4 levels, high normal FT3, and TSH levels are associated with less favorable lipid parameters. BMI modulates the effect of thyroid hormones on lipid metabolism. The findings that the associations between $\mathrm{TH}$ and lipid parameters are not identical in normal-weight and overweight/obese subjects provide clues for individualized lipid-lowering therapy in clinic.

\section{Conflicts of Interest}

The authors declare that there is no conflict of interest regarding the publication of this article.

\section{Authors' Contributions}

Yanqiu Wang, Qinglei Yin, Min Xu, and Qicheng Ni performed the experiments and analyses. Yanqiu Wang and 
Qidi Wang wrote the manuscript. Weiqing Wang and Qidi Wang designed the project, supervised the research, and coordinated the execution of the experimental plan. Yanqiu Wang and Qinglei Yin contributed equally to this work.

\section{Acknowledgments}

This work was supported by the National Natural Sciences Foundation of China Grants (81370875 and 81670700) and the National Basic Research Program of China (2015CB553601). The authors thank M. Zhang (Department of Nuclear Medicine, Ruijin Hospital) for collecting the clinical data.

\section{References}

[1] C. J. L. Murray and A. D. Lopez, "Mortality by cause for eight regions of the world: global burden of disease study," The Lancet, vol. 349, no. 9061, pp. 1269-1276, 1997.

[2] Y. Chen, X. Wu, R. Wu et al., "Changes in profile of lipids and adipokines in patients with newly diagnosed hypothyroidism and hyperthyroidism," Scientific Reports, vol. 6, article 26174, 2016.

[3] R. Mullur, Y.-Y. Liu, and G. A. Brent, "Thyroid hormone regulation of metabolism," Physiological Reviews, vol. 94, no. 2, pp. 355-382, 2014.

[4] G. A. Brent, "Graves' disease," New England Journal of Medicine, vol. 358, no. 24, pp. 2594-2605, 2008.

[5] G. A. Brent, "Mechanisms of thyroid hormone action," The Journal of Clinical Investigation, vol. 122, no. 9, pp. 30353043.

[6] G. L. Roef, E. R. Rietzschel, C. M. Van Daele et al., "Triiodothyronine and free thyroxine levels are differentially associated with metabolic profile and adiposity-related cardiovascular risk markers in euthyroid middle-aged subjects," Thyroid, vol. 24, no. 2, pp. 223-231, 2014.

[7] A. Roos, S. J. L. Bakker, T. P. Links, R. O. B. Gans, and B. H. R. Wolffenbuttel, "Thyroid function is associated with components of the metabolic syndrome in euthyroid subjects," The Journal of Clinical Endocrinology \& Metabolism, vol. 92, no. 2, pp. 491-496, 2007.

[8] S. Temizkan, B. Balaforlou, A. Ozderya et al., "Effects of thyrotrophin, thyroid hormones and thyroid antibodies on metabolic parameters in a euthyroid population with obesity," Clinical Endocrinology, vol. 85, no. 4, pp. 616-623, 2016.

[9] F. Wang, Y. Tan, C. Wang et al., “Thyroid-stimulating hormone levels within the reference range are associated with serum lipid profiles independent of thyroid hormones," The Journal of Clinical Endocrinology \& Metabolism, vol. 97, no. 8, pp. 2724-2731, 2012.

[10] J. Bassols, A. Prats-Puig, P. Soriano-Rodríguez et al., "Lower free thyroxin associates with a less favorable metabolic phenotype in healthy pregnant women," The Journal of Clinical Endocrinology \& Metabolism, vol. 96, no. 12, pp. 3717-3723, 2011.

[11] Y. Zhang, P. Lu, L. Zhang, and X. Xiao, “Association between lipids profile and thyroid parameters in euthyroid diabetic subjects: a cross-sectional study," BMC Endocrine Disorders, vol. 15, p. 12, 2015.
[12] J. Wang, X. Zheng, M. Sun et al., "Low serum free thyroxine concentrations associate with increased arterial stiffness in euthyroid subjects: a population-based cross-sectional study," Endocrine, vol. 50, no. 2, pp. 465-473, 2015.

[13] M. Laclaustra, Y. Hurtado-Roca, M. Sendin et al., "Lower-normal TSH is associated with better metabolic risk factors: a cross-sectional study on Spanish men," Nutrition, Metabolism and Cardiovascular Diseases, vol. 25, no. 12, pp. 1095-1103, 2015.

[14] J. J. Lee, A. Pedley, E. Marqusee et al., "Thyroid function and cardiovascular disease risk factors in euthyroid adults: a cross-sectional and longitudinal study," Clinical Endocrinology, vol. 85, no. 6, pp. 932-941, 2016.

[15] H. S. Shon, E. D. Jung, S. H. Kim, and J. H. Lee, "Free T4 is negatively correlated with body mass index in euthyroid women," The Korean Journal of Internal Medicine, vol. 23, no. 2, pp. 53-57, 2008.

[16] P. Marzullo, C. Mele, S. Mai et al., "The impact of the metabolic phenotype on thyroid function in obesity," Diabetology \& Metabolic Syndrome, vol. 8, no. 1, p. 59, 2016.

[17] A. Nagel, A. Spinneker, and M. Neuhäuser-Berthold, “Association of thyroid-stimulating hormone with resting energy expenditure in euthyroid elderly subjects: a cross-sectional study," Annals of Nutrition and Metabolism, vol. 68, no. 1, pp. 12-18, 2016.

[18] B. O. Åsvold, T. Bjøro, and L. J. Vatten, “Association of serum TSH with high body mass differs between smokers and neversmokers," The Journal of Clinical Endocrinology \& Metabolism, vol. 94, no. 12, pp. 5023-5027, 2009.

[19] J. J. Díez and P. Iglesias, "Relationship between thyrotropin and body mass index in euthyroid subjects," Experimental and Clinical Endocrinology \& Diabetes, vol. 119, no. 03, pp. 144-150, 2011.

[20] X. Wang, H. Liu, J. Chen et al., "Metabolic characteristics in obese patients complicated by mild thyroid hormone deficiency," Hormone and Metabolic Research, vol. 48, no. 5, pp. 331-337, 2016.

[21] World Health, O, "Physical status: the use and interpretation of anthropometry," WHO Technical Report Series, vol. 0, no. 854, 1995.

[22] B. O. Åsvold, T. Bjøro, C. Platou, and L. J. Vatten, “Thyroid function and the risk of coronary heart disease: 12-year follow-up of the HUNT study in Norway," Clinical Endocrinology, vol. 77, no. 6, pp. 911-917, 2012.

[23] Y. K. Lee, J. E. Kim, H. J. Oh et al., "Serum TSH level in healthy Koreans and the association of TSH with serum lipid concentration and metabolic syndrome," The Korean Journal of Internal Medicine, vol. 26, no. 4, pp. 432-439, 2011.

[24] S. Galic, J. S. Oakhill, and G. R. Steinberg, "Adipose tissue as an endocrine organ," Molecular and Cellular Endocrinology, vol. 316, no. 2, pp. 129-139, 2010.

[25] T. Reinehr, "Obesity and thyroid function," Molecular and Cellular Endocrinology, vol. 316, no. 2, pp. 165-171, 2010.

[26] B. Klop, J. W. F. Elte, and M. Castro Cabezas, "Dyslipidemia in obesity: mechanisms and potential targets," Nutrients, vol. 5, no. 4, pp. 1218-1240, 2013.

[27] F. Santini, P. Marzullo, M. Rotondi et al., "Mechanisms in endocrinology: the crosstalk between thyroid gland and adipose tissue: signal integration in health and disease," European Journal of Endocrinology, vol. 171, no. 4, pp. R137-R152, 2014. 
[28] M. Yamamoto, K. Uchihashi, S. Aoki, E. Koike, N. Kakihara, and S. Toda, "Interaction between thyrocytes and adipose tissue in vitro," Pathology International, vol. 66, no. 3, pp. 148-157, 2016.

[29] P. Kok, F. Roelfsema, J. G. Langendonk et al., "High circulating thyrotropin levels in obese women are reduced after body weight loss induced by caloric restriction," The Journal of Clinical Endocrinology \& Metabolism, vol. 90, no. 8, pp. 4659-4663, 2005. 


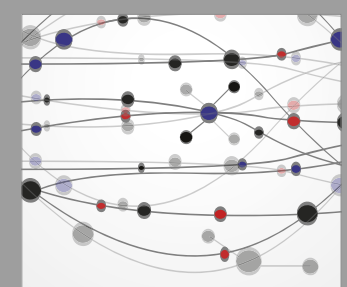

The Scientific World Journal


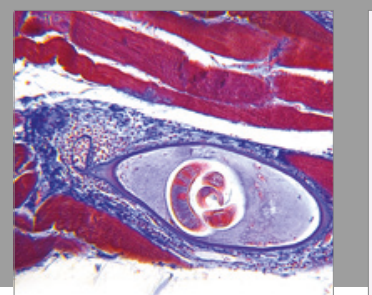

Gastroenterology Research and Practice
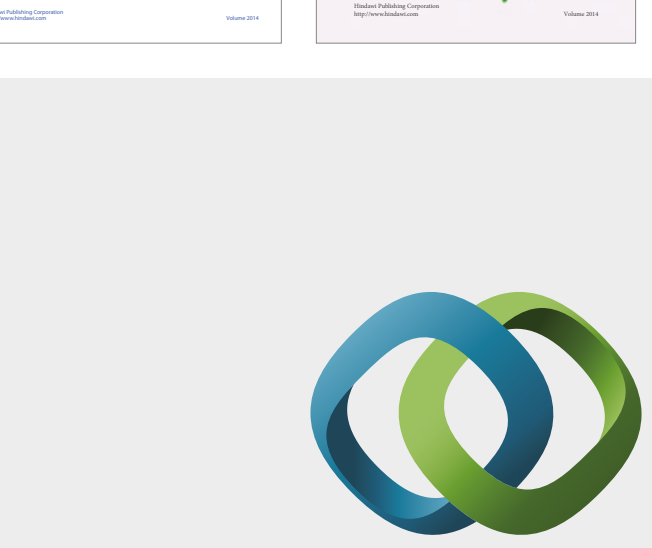

\section{Hindawi}

Submit your manuscripts at

https://www.hindawi.com
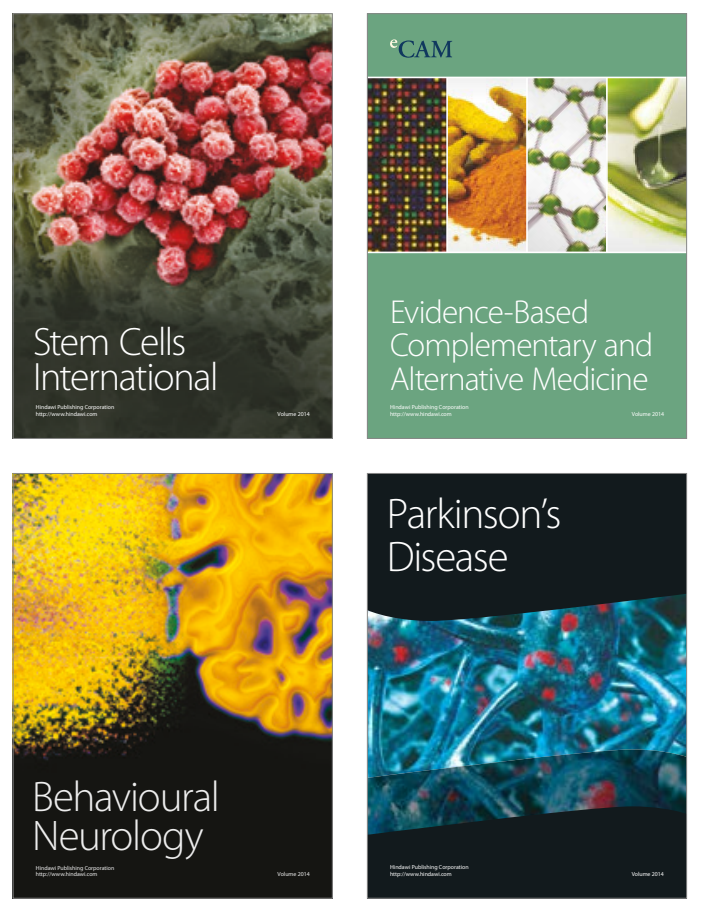
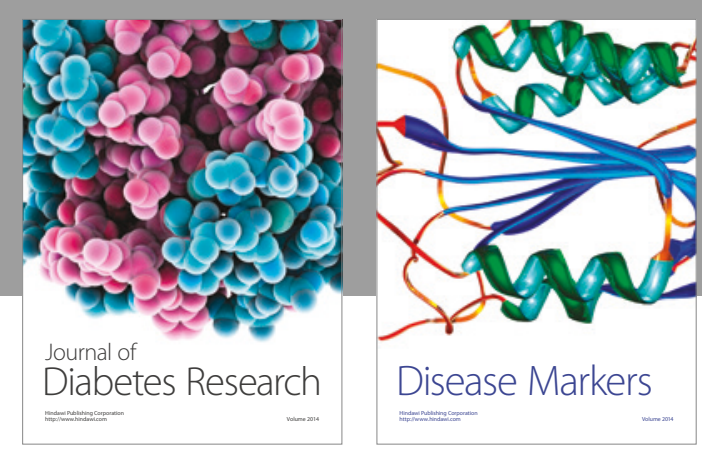

Disease Markers
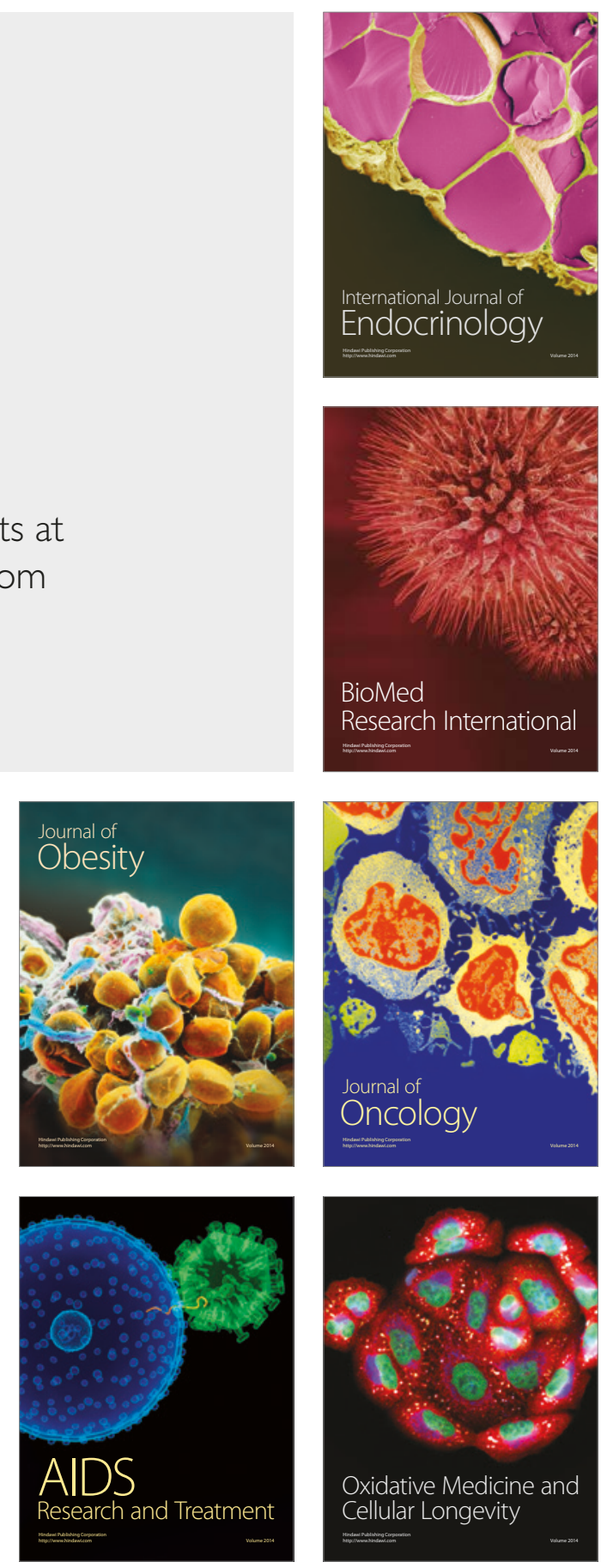